\title{
Tabellen- und Abbildungsverzeichnis
}

\section{Tabellen}

Tabelle 4.1 Kontingenztafel, Gegenüberstellung der Häufigkeitsverteilungen der Anzahl der E-Mails mit und ohne NS-V/M in Bezug auf die uV (Institution: IBD vs. $\mathrm{Zd})$ ) 181

Tabelle 4.2 $X^{2}$ nach Pearson -182

Tabelle 4.3 Phi und Cramers V 182

Tabelle 4.4 Kontingenztafel, Gegenüberstellung der Häufigkeitsverteilungen der aV (Anzahl der E-Mails mit und ohne NS-V/M) in Bezug auf die uV (Zeitraum: Konfliktzeitraum vs. kein Konfliktzeitraum) -187

Tabelle 4.5 $X^{2}$ nach Pearson -188

Tabelle 4.6 Phi und Cramers V -188

Tabelle 4.7 Auflistung der Verteilung soziodemografischer Merkmale der Verfasser*innen von E-Mails mit NS-V/M -190

Tabelle 5.1 Absolute und relative Häufigkeitsverteilung von NS-V/M im Korpus nach Art der Realisierung — 198

Tabelle 5.2 Absolute und relative Häufigkeitsverteilung der sprachlichen Realisierungsformen von NS-V im Korpus - 199

Tabelle 5.3 Absolute und relative Häufigkeitsverteilung der lexikalischen Vergleichsindikatoren sowie des NS-Vokabulars - 210

Tabelle 5.4 Absolute und relative Häufigkeitsverteilung der NS-M nach konzeptuellem Ursprungsbereich -245

Tabelle 5.5 Absolute und relative Häufigkeit der Komparationsbasen und konzeptuellen Ursprungsbereiche der NS-V/M -248

Tabelle 5.6 Absolute und relative Häufigkeit der dominanten antisemitischen Stereotype in E-Mails mit NS-V/M -283

Tabelle A1 Korpus: Anzahl der E-Mails - 337

Tabelle A2 Sprachliche Realisierung der NS-V/M - 338

Tabelle A3 Komparationsbasen und metaphorische Ursprungsbereiche der NS-V/M — 339

Tabelle A4 Komparanden und metaphorische Zielbereiche der NS-V/M - $\mathbf{3 4 0}$

Tabelle A5 Codesystem zur qualitativen Analyse der E-Mails mit NS-V $/ \mathrm{M}^{\dagger}-\mathbf{3 4 1}$

\section{Abbildungen}

Abbildung 4.1 Absolute Häufigkeitsverteilung der E-Mails mit und ohne NS-V/M an die IBD im gesamten Jahresverlauf (Konfliktzeiträume und keine Konfliktzeiträume inbegriffen) - 186

Abbildung 4.2 Relative Häufigkeitsverteilung der E-Mails mit NS-V/M an die IBD im Verlauf der Jahre (Konfliktzeiten und keine Konfliktzeiten inbegriffen) -187 
